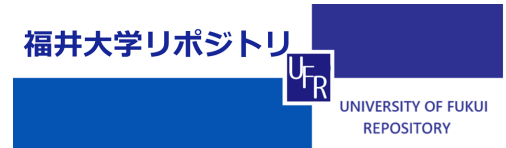

The Effect of the Nonuniformty of the Nagnet i c Fi el d on the Beam Pl asma I nst abi I i ty

\begin{tabular}{|l|l|}
\hline $\begin{array}{l}\text { j our nal or } \\
\text { publ i cat i on t i t l e }\end{array}$ & 福井大学工学部研究報告 \\
\hline vol une & 26 \\
\hline number & 2 \\
\hline page $r$ ange & $155-165$ \\
\hline year & 1978 - 09 \\
\hline URL & ht t p: //hdl . handl e. net /10098/4448 \\
\hline
\end{tabular}


MEMOIRS OF THE FACULTY OF ENGINEERING
FUKUI UNIVERSITY

$\begin{array}{lll}\text { VOL.26 No.2 } & 1978\end{array}$

\section{The Effect of the Nonuniformity of the Magnetic Field on the Beam-Plasma Instability}

T. Idehara, M. Tanaka ${ }^{*}$ and Y. Ishida*

The experimental investigation of the space charge wave instability of the spiral beam-plasma system in a nonuniform magnetic field is reported. The velocity distribution of spiral electron beam is varied by the beam accerelation effect resulting from the parallel force due to the negative gradient of field intensity, which affects the trapping of beam electrons on the potential of the unstable wave. As the results, it is observed that the saturation length and the saturation level of the wave become extremely large. The brief theoretical analysis can explain the above experimental results, consistently.

The interaction of the particles in plasma with the monochromatic plasma wave has been investigated with a great interest by many authors. ${ }^{1}$ ) As well-known, the particles trapped in a trough of the wave potential does play a main part, which are bunched in both real and velocity spaces and as

* Department of Applied Physics 
the result, the wave amplitude is observed to oscillate about a value of the metastable state with the bounce frequency of them. ${ }^{2)}$ Drumond et al. ${ }^{3 \text { ) }}$ show that the similar behavior occurs in a monoenergetic beam-plasma system, where a narrow-band unstable wave grows monotonically at a linear growth rate, until the beam particles are trapped and the wave amplitude begins to oscillate as the same manner as mentioned above. ${ }^{4)}$ The experimental study of the spatial development of the instability in a beam-plasma system has justified the above theoretical considerations. ${ }^{5)}$

In this letter, we report an experimental study of the spiral beamplasma system in a nonuniform magnetic field, designed to understand the effect of the trapping of beam particles on the instability of plasma wave. The velocity distribution of electron beam is controlled by parallel force due to the gradient of field intensity, which affects the trapping of beam electrons. Traveling in the region where the field intensity decreases, they are accerelated in the direction parallel to the field and not trapped until the wave amplitude becomes so large that the potential energy of wave exceeds that of beam electrons containing the additional energy due to the accerelation effect. Then, the saturation length and the saturation level of the wave become extremely larger than those of the case of uniform magnetic field.

The plasma produced by the TP-D plasma source ${ }^{6)}$ diffuses along the line of magnetic force into the region of the beam-plasma system, which consists of the pylex glass tube $(9.5 \mathrm{~cm}$ in diameter and $72 \mathrm{~cm}$ in length). Its parameters are as follows. The density $n_{p}$ at the center of the tube is changed from $1.3 \times 10^{9}$ to $1.0 \times 10^{10} \mathrm{~cm}^{-3}$ by varying the discharge 
current $I_{d}$ of plasma source, and its profile in the axial direction is uniform within about 5 percent. The electron temperature $\mathrm{T}_{\mathrm{e}}=7.0 \mathrm{eV}$, the plasma diameter $D=30 \mathrm{~mm}$ and the pressure of the neutrals (Ar) $p=7.4$ $\times 10^{-4}$ Torr (the collision frequency of electron with neutrals $\nu_{\text {en }}=4 \mathrm{MHz}$ ). An electron beam is generated by the Pierce gun placed on the opposite side to the plasma source and injected into the beam-plasma region through an orifice whose diameter and thickness are $15 \mathrm{~mm}$ and $10 \mathrm{~mm}$, respectively. The gun is set on the tube axis and shoots the beam obliquely to the line of magnetic force, so that the spiral beam is formed. By varying the shooting angle $\theta$ and the accerelating voltage $V_{b}$, the ratio $E_{\perp} / E_{b}$ of the energy component perpendicular to the field to the total energy of beam is controlled within the region from 0 to 50 percent and $\mathrm{E}_{\mathrm{b}}$ is done from 50 to $300 \mathrm{eV}$. When the cathode current of gun changes from 3 to $20 \mathrm{~mA}$, the beam density $n_{b}$ is varied from $1.5 \times 10^{8}$ to $4.5 \times 10^{8} \mathrm{~cm}^{-3}$, but its temperature $\mathrm{T}_{\mathrm{b}}$ is constant at about $0.3 \mathrm{eV}$. The magnetic field is applied along the tube axis by ten coils. Its intensity distribution on the axis is varied as shown in Figs. $I$ and 2 , by varying the current $I_{2}$ of six coils on the right hand side and keeping the current $I_{l}$ of the other four coils constant at $1.0 \mathrm{~A}$.

In order to excite and receive the wave, five coaxial probes are inserted in the beam-plasma region, three of them being movable radially ( $r$-probe) and the other two being movable axially (z-probe). The signal of the wave excited by a probe is detected using another probe and recorded by the interferometer system. This apparatus has been used in our experiment reported in the previous monogragh. ${ }^{\text {) }}$ 


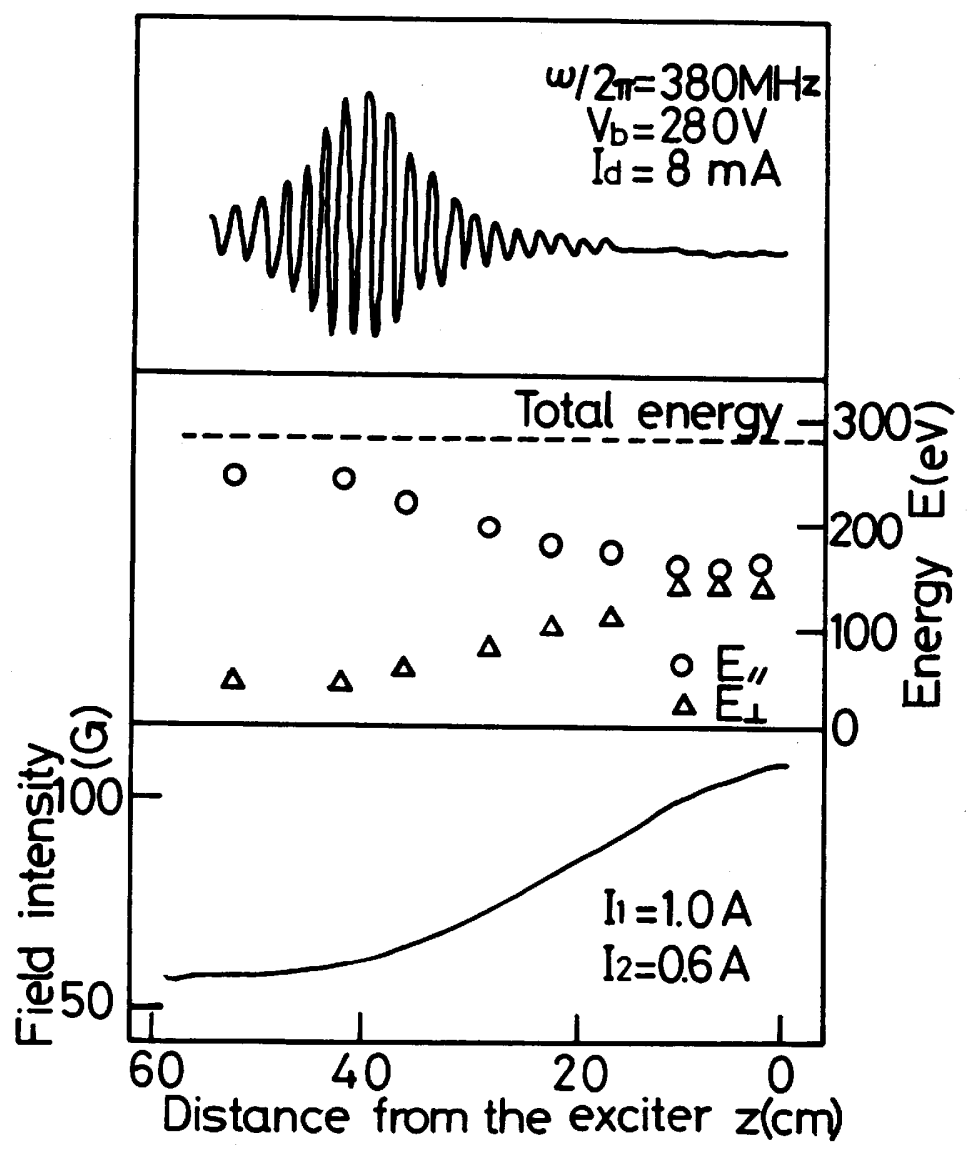

Fig. I The lower trace; The profile of the external magnetic field intensity along the tube axis. The middle one; The variation of the energy components $\left(E_{\perp}\right.$ and $\left.E_{11}\right)$ of the spiral electron beam in the absence of the plasma. The beam inlet is situated at the point of $z=-3 \mathrm{~cm}$. The upper one; The propagating wave patterm in the spiral beam-plasma system. 
In Fig. 1, are shown the typical experimental result of the variation of the beam energy components in the nonuniform magnetic field and the propagating pattern of the externally excited test wave. First, the behavior of the electron beam in the case where the nonuniform magnetic field is applied, is studied in the neutral Ar gas. The electron beam whose energy component $E_{\perp}$ is about 50 percent of the total energy $E_{b}$ is injected from the right hand side in the figure. The accerelation parallel to the field occurs, because of the parallel force $F_{11}(=-\mu \nabla B)$ applied from the gradient of field intensity. By measuring the variation of the electron current of the axially movable probe, the pitch $p$ of spiral beam and the energy component $E_{11}=m \omega_{c}^{2} p^{2} / 8 \pi^{2}$ are determined, the latter being shown as a function of $z$ in Fig. I, together with the other component $E_{\perp}=E_{b}-E_{\| 1}$. $m$ and $\omega_{c} / 2 \pi$ denote the electron mass and the electron cyclotron frequency. It is known that the magnetic moment $\mu=E_{\perp} / B$ of beam electron is kept nearly constant at $\mu=1.2 \pm 0.3 \mathrm{eV} /$ gauss.

Next, the propagation of the test wave is studied in the plasma penetrated by the spiral beam mentioned above, which is shown in the upper trace of Fig. 1. It is seen that the wave launched by the exciting probe situated at $\mathrm{z}=0$ continues to be amplified in the region where the parallel accerelation of beam occurs, that is, in the region where the gradient of the field intensity has an appreciable value. This wave is considered to be a space charge wave of beam, because it satisfies the Cherenkov condition.

The propagating wave patterns are observed in various configurations of field intensity for the same initial condition of injected eletron beam as the case of Fig. I, the results of which and the corresponding field 


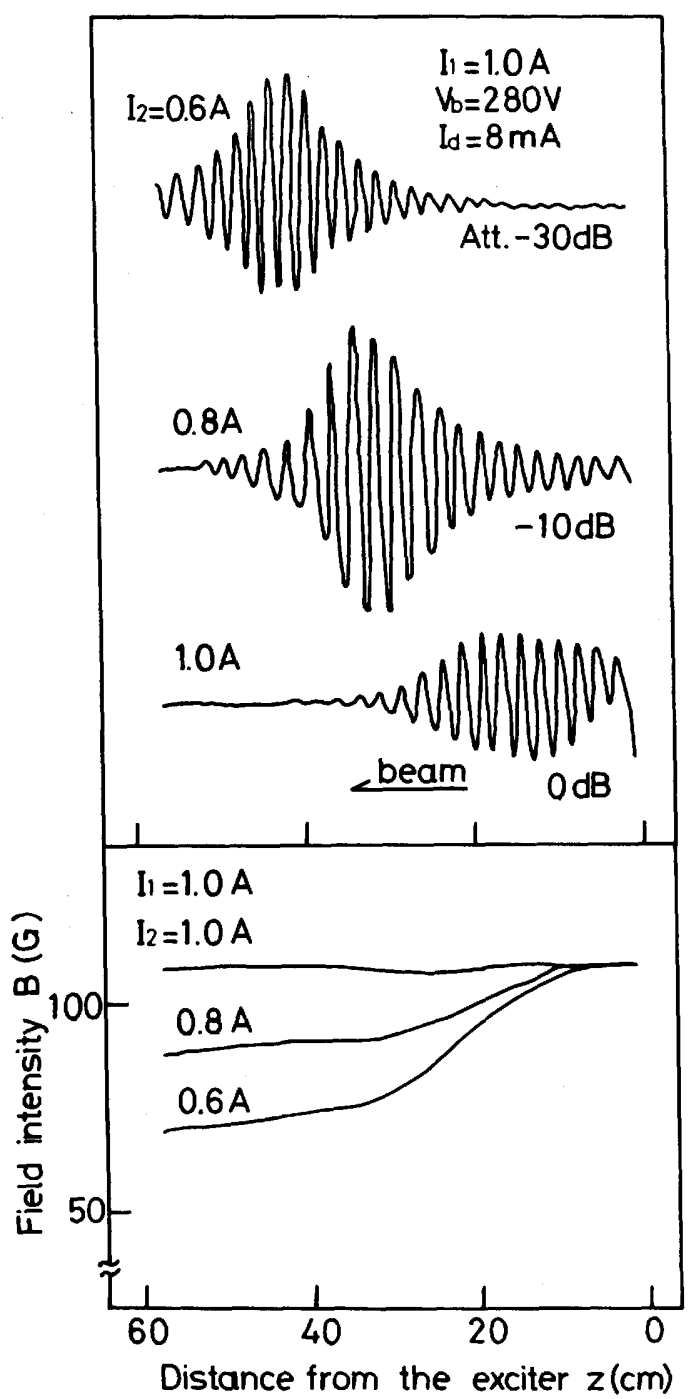

Fig. 2 The lower traces; Various profiles of the external magnetic field intensity along the tube axis. The upper traces; The propagating wave patterms corresponding to each profile of field intensity, in the spiral beam-plasma system. 
configurations being shown in Fig. 2. As the amplification of the wave occurs through the region of a nonuniform magnetic field, the saturation length $z_{S}$ and the saturation level $\tilde{E}^{2}$ in the case of nonuiform field become extremely larger than those $\left(z_{0}\right.$ and $\left.\widetilde{E}_{0}^{2}\right)$ of the case of a uniform field $\left(I_{2}=1.0 \mathrm{~A}\right.$ ). (It must be noted that the sensitivity of measuring system is lower than that of the latter case by several ten dB.)

The saturation length $z_{S}$ and the normalized saturation level $\tilde{\mathrm{E}}^{2} / \tilde{\mathrm{E}}_{0}^{2}$ are plotted in Fig. 3 as functions of the coil current $I_{2}$ which is a parameter of the nonuniformity of field. Though the real value of the field intensity of wave cannot be measured, the value of $\tilde{\mathrm{E}}^{2} / \tilde{\mathrm{E}}_{0}^{2}$ is sufficiently significant because the frequency of the test wave is constant through the experiment and we may assume that the coupling constant of the probe to the plasma does not vary.

The results can be considered as follows. The saturation of the wave amplitude occurs, when the potential $\tilde{\phi}$ of the wave grows and becomes so large that the beam electrons begion to be trapped on its trough. The initial energy component $E_{110}=\frac{1}{2} m_{110}^{2}$ of the beam electron in the wave frame is related to the saturation level $\tilde{E}_{0}^{2}$ in the case of a uniform magnetic field as follows,

$$
E_{110}=\left|e \tilde{E}_{0} / k\right|=\left|e \tilde{\phi}_{0}\right|
$$

where $e$ and $k$ denote the electric charge and the wave number of growing wave. On the other hand, in the case of a nonuriform field, 


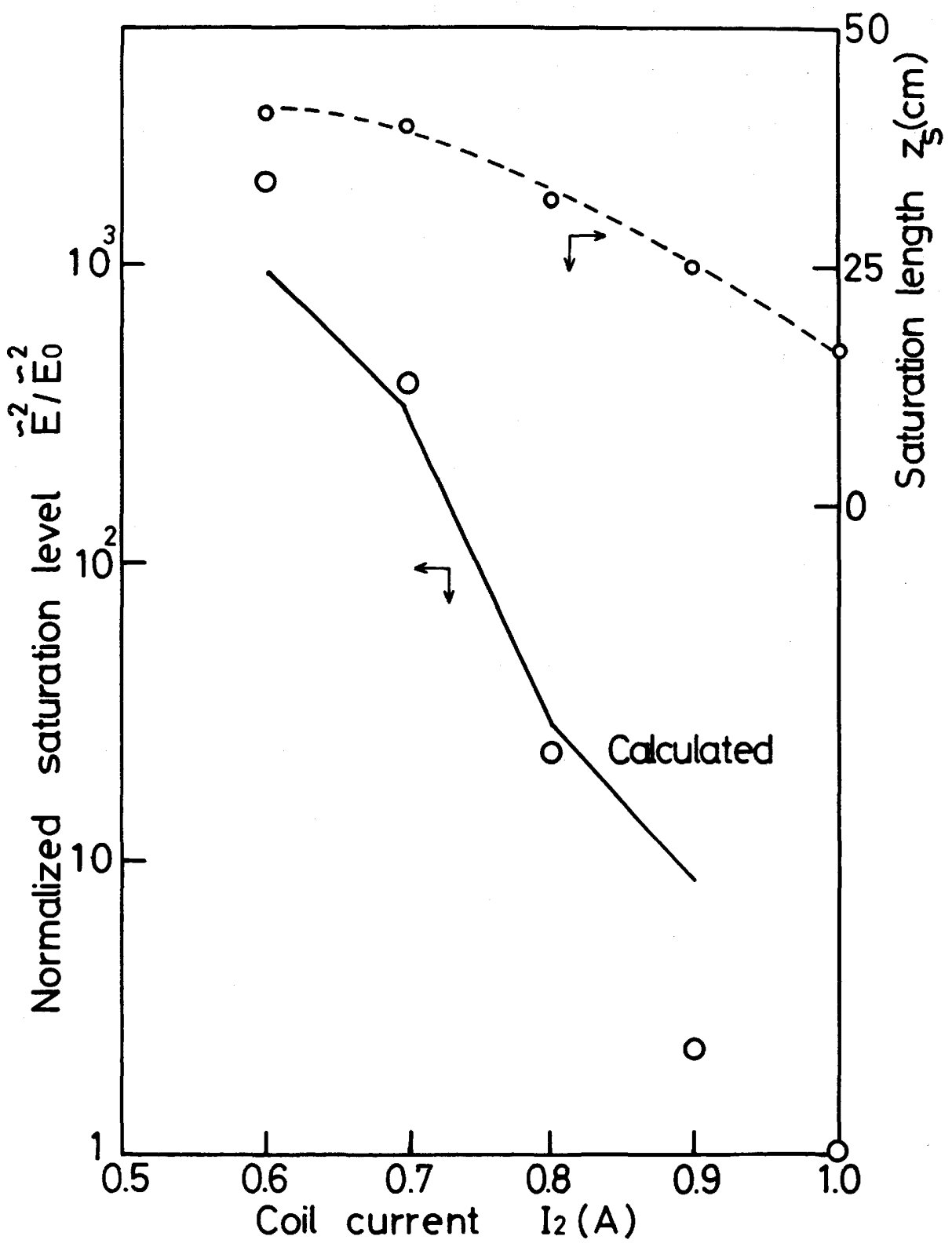

Fig. 3 The saturation length $z_{S}$ and the normalized saturation level $\tilde{E}^{2} / \tilde{E}_{0}^{2}$ as functions of the coil current $I_{2}$. The solid line shows the result of calculation for $\tilde{\mathrm{E}}^{2} / \tilde{E}_{0}^{2}$. 


$$
E_{110}+\mu \Delta B\left(z=z_{S}\right)=|e \tilde{E} / k|
$$

where we define the decrease of field intensity at the saturation point as $\Delta B\left(z=z_{S}\right)$. Therefore, the second term of the left hand side of eq. (2) shows an additional beam energy obtained from the gradient of field intensity before the beam electrons begin to be trapped. The normalized saturation level is expressed as follows,

$$
\frac{\tilde{E}^{2}}{\tilde{E}_{0}^{2}}=\left(\frac{E_{110}+\mu \Delta B\left(z=z_{s}\right)}{E_{10}}\right)^{2}
$$

By using the experimentally obtained value of $\mu(=1.2 \mathrm{eV} /$ gauss $)$ and the value of $\Delta B\left(z=z_{S}\right)$ given by the field intensity at the saturation point for each configuration, we calculate the normalized saturation level, which is plotted by a solid curve in Fig. 3. Here, $E_{110}$ is assumed to be constant at $2.0 \mathrm{eV}$. The calculated values show the fairly good agreement with the experimental results.

The energy loss of electron beam due to the energy transfer to the wave results in the decrease $\Delta p$ of the pitch of it. The detailed measurement of $\Delta p$ shows that the value of $E_{110}$ is about $5.0 \mathrm{eV}$. This value is defferent by only factor 2.5 from that assumed above. Therefore, the result of calculation may explain the experimental results consistently. 
In conclusion, we have verified the following facts; (1) the spiral electron beam is accerelated parallel to the magnetic field by the force due to the gradient of the field intensity, (2) the test wave launched in the spiral beam-plasma system continues to be amplified through the region where the beam is accerelated, so that the saturation level of the wave becomes extremely larger than that of the case in a uniform field, and (3) these results are explained consistently by the brief consideration of trapping of beam electrons on the trough of wave potential. These facts may suggest the possibility of the effective energy transfer from the electron beam to the plasma in a proper configuration of magnetic field.

This work is partially supported by a Grant-in-Aid from the Ministry of Education in Japan.

References

1) For instance, R.Z. Sagdeev and A.A. Galeev, in Nonlinear Plasma Theory, edited by T. O'Neil and D. Book (Benjamin, New York, 1969) p.37. T. O'Neil, Phys. of Fluids 8 (1965) 2255.

2) J.H. Malmberg and C.B. Wharton, Phys. Rev. Letts. 19 (1967) 775. N. Sato, H. Ikezi, N. Takahashi and Y. Yamashita, Phys. Rev. 183 (1969) 278 .

3) W.E. Drummond, J.H. Malmberg, T. O'Neil and J.R. Thompson, Phys. of Fluids 13 (1970) 2422.

4) J.R. Thompson, Phys. of Fluids 14 (1971) 1532. 
5) J.H. Malmberg and C.B. Wharton, Phys. of Fluids 12 (1969) 2600. K. Mizuno and S. Tanaka, Phys. Rev. Letts. 29 (1972) 45.

6) K. Takayama, M. Ohtsuka, Y. Tanaka, K. Ishii and Y. Kubota, in Proc. of 7 th Intern. Conf. on Phenomena in Ionized Gases (Gradevinska, Kujiga, Beograd, Yugoslavia, 1966) Vol. I, p. 420.

7) T. Idehara, N. Miyama and Y. Ishida, J. Phys. Soc. Japan 42 (1977) 1730. 
